with true adsorption and desorption processes. The amount of water adsorbed at 100 per cent relative humidity is not indicated because of the difficulty in preventing actual condensation of water on the wool. In every instance the amount of water adsorbed by the wool which had been stretched was greater than that adsorbed by the untreated sample, and it seems clear that plastic flow in the fibrillar structure alone will increase the affinity of wool for water.

J. B. SPEAKMAN. The University,
Leeds,

Aug. 14.

\section{The Adsorption of Organic Acids by Charcoal.}

IN the recent issue of the Proceedings of the Royal Society (A, 124, 554; 1929), H. J. Phelps and R. A. Peters, while studying the adsorption of some organic acids on pure charcoal as a function of the hydrogen ion concentration, come to the conclusion that, in the given conditions, the non-dissociated molecules and not the corresponding anions are adsorbed. This conclusion is based on similar observations to those already made by $R$. Wurmser and myself some years ago $(C . R ., 179,972 ; 1924)$ with another charcoal, that, while for different acids there is no relation between adsorption and dissociation, in the case of the same acid, there is a very definite relation between these two phenomena; and I am very glad to see our results confirmed and enlarged by the interesting work of Messrs. Phelps and Peters.

May I now recall that, in another communication published in the same year (C.R., 179, 1404; 1924), which seems to have escaped the attention of the authors, I was able to give a definite proof of the selective adsorption of non-dissociated molecules by the charcoal used. Having previously determined (spectrographically) the sum molecules + possible anions adsorbed, I measured the electrical charge acquired by the charcoal particles after adsorption, and found it completely independent of the quantity and of the nature of the adsorbed acid, the data being of course reduced to the same hydrogen ion concentration. It seems, therefore, that this is the best proof of the electrical neutrality of the adsorbed substance.

It is easy to see that this result, of course, does not exclude the possibility of electric forces playing a rôle in that type of adsorption phenomena, when one recalls that the molecules adsorbed here are dipoles which are liable to be affected by electrostatic forces (cf. V. Henri, "Structure des molécules").

Claude Fromageot.

Kaiser Wilhelm Institut für Biochemie, Berlin-Dahlem.

\section{Anomalous Diamagnetism.}

IN a letter in NaTuRE of June 22 (p. 945), reference was made to the Ehrenfest hypothesis which ascribes the high diamagnetic susceptibility of bismuth to the existence of closed electron orbits of larger than atomic dimensions in association with the crystal lattice.

It may be pointed out that the existence of such orbits appears to furnish a very natural explanation of a variety of phenomena which up to now have been obscure. In the first place, the extremely pronounced diamagnetic anisotropy characteristic of bismuth (and also of graphite) becomes immediately intelligible as a consequence of the specific orientation of the assumed electronic circulations within the crystal lattice. Further, the large Hall effects and changes of electrical resistance exhibited by bismuth and graphite when placed in a magnetic field become comprehensible, since the electronic circulations would be modified by the field, and result in corresponding modifications of the flow of electricity through the substance under a simultaneously impressed electromotive force. There would be every reason to expect, as is indeed the case, that the magnitudes of the Hall effect and the change of resistance would depend on the orientation of the crystal in the magnetic field and the direction of flow of electricity through it.

Then again, with rise of temperature and consequent thermal derangements of the lattice, the postulated electronic circulations would tend to disappear and give place to chaotic electronic movements. The diamagnetic susceptibility would then diminish towards its normal value for a non-crystalline condition of the substance, and corresponding changes would occur in the coefficients of the Hall effect and magnetic variation of electrical resistance. That liquid metals do not, so far as I am aware, exhibit a measurable Hall effect is significant in this connexion.

In close analogy with the influence of tempera. ture, and presumably to be explained on very similar lines, is the remarkable fact that the anomalous diamagnetism of bismuth and of graphite tends to diminish or disappear when the substances are reduced to a colloidal condition.

Finally, it may be remarked that the dimensions of the erystal lattice cannot be uninfluenced by the existence of such regular electronic circulations within it or by modifications produced in them by an external agency. That the magnetostriction of bismuth in strong fields discovered by Dr. Kapitza (NATure, July 13, p. 53) is connected with the anomalous diamagnetism of the substance admits of little doubt. The notable increase in magnetostriction at low temperatures observed by him appears to fit in very well with the Ehrenfest hypothesis.

\section{V. RAMaN}

\section{Dew : Does it Rise or Fall ?}

Messns. E. E. Free and Travis Hoke begin Chapter xiii. of their recent work on "Weather" (Constable and Co., 1929) by stating that "the chief thing to be said about dew is that it doesn't fall, early or late, on Maxwelton or any other braes. In fact it rises." They go on to explain that dew is formed when the warm, moist "breath of the ground" comes in contact with colder objects, such as leaves, twigs, or spider webs, causing precipitation. They make no note of the fact that dew is formed only under a clear sky which allows free radiation of the earth's surface heat; but they make the following surprising statement: "Most of the dewdrops on leaves and blades of grass, especially leaves close to the ground, are on the under sides; that is, the side of the leaf or grass closest to the soil."

This was so widely at variance with the general impression I had received, that I went out on three dewy mornings to examine the herbage, and found, as I expected, that while the upper surfaces of leaves were heavily bedewed, the under surfaces were quite dry.

In stating that dew does not fall, but rises, the authors seem to be treating of dew before it is formed. It is true that the warm air radiated from the earth is charged with moisture, but that continues as vapour until it meets the cold night or morning air, when it is condensed and precipitated as dew. The vapour rises, but the dew can only be described as falling.

Monreith.
Herbert Maxwell.

No. 3124, VoL. 124] 